\title{
Quantum Simulation of SiC Nanotubes
}

\author{
Qiyin Luo ${ }^{1}$, Mitsuhiro Matsumoto ${ }^{1}$ \\ ${ }^{1}$ Graduate School of Engineering, Kyoto University \\ Kyoto-Daigaku-Katsura, Kyoto 615-8540, Japan \\ luoqiyin2012@gmail.com; matsumoto@kues.kyoto-u.ac.jp
}

\begin{abstract}
We performed a series of quantum simulations for the geometrics, electronic structures and electron transport of various types of silicon carbide nanotubes (SiCNT's). The 1:1 SiCNT's generally show more stable semi-conducting behavior than carbon nanotubes (CNT's). The SiCNT's exhibit better electronic properties than their bulk materials. Their band gap increases with increasing the tube diameter. Armchair SiCNT's have narrower band gap than zigzag ones, whereas the zigzag SiCNT's possess direct band gap.
\end{abstract}

Keywords: Silicon carbide nanotubes, Quantum simulation, Electronic structures, Band gap, Electron transport

\section{Introduction}

The discovery of carbon nanotubes (CNT's) has aroused a tremendous explosion in general interest in quasi-onedimensional nanomaterials because of their potential use in various nanotechnology and nanoscale engineering. As for their application in semiconductor fields, the electronic properties depend on their chirality. There has been much expectation that other materials, such as silicon-based ones, with similar nano-scale structures have better semiconducting properties.

Among the various types of semiconducting materials, silicon carbide possesses unique physical and electronic properties, and its application to electronic devices has been pursued, such as devices for high-temperature, high-power, and high-frequency conditions. Since the success in synthesis of boron nitride nanotubes [1-3], various types of low-dimensional materials of non-carbon or partial carbon compounds have been explored. Two-dimensional (graphitic) silicon is found to be unstable [4], for example. However, $\mathrm{C}-\mathrm{C}$ and $\mathrm{Si}-\mathrm{C}$ bonds are known to be more stable than the Si-Si bond, which can be utilized to stabilize Si nanotubes [5]. Also some types of silicon carbide nanotubes (SiCNT's) have electronic properties much different from their bulk materials.

In this paper, we systematically explore the electronic properties of various SiCNT's using quantum simulations.

\section{Method}

We adopted the density functional tight binding (DFTB) model for the quantum simulation to evaluate the structure, electronic properties, and electron transport behaviors of SiCNT's. The DFTB+ package [6] was used.

A self consistent charge (SCC) calculation was made with the tolerance of charge fluctuations $10^{-7}$. The periodic boundary condition is set along the tube axis $(z)$, while the vacuum space of $30 \AA$ is assumed along the radial directions to evaluate the properties of single tube. The supercells contains 16 layers of atoms along the tube axis for both armchair and zigzag types. The number of $k$ points is chosen to be $(1 \times 1 \times 4)$.

For each SiCNT, we performed a molecular dynamics (MD) calculation with temperature control; after constructing initial atomic configuration, we gradually raised the temperature to look into the mechanical stability. In this paper, results at $300 \mathrm{~K}$ are mainly reported. The average length of Si-C bonds was about $1.80 \AA$, in agreement with Ref. [5].

\section{Results and Discussion}

Various types of SiCNT's with different diameters and chiralities (zigzag-type or armchair-type) were prepared, where $\mathrm{Si}$ and $\mathrm{C}$ atoms are placed alternatively without any adjacent atoms of the same element. After equilibrating the system at $300 \mathrm{~K}$, electronic properties were investigated. 

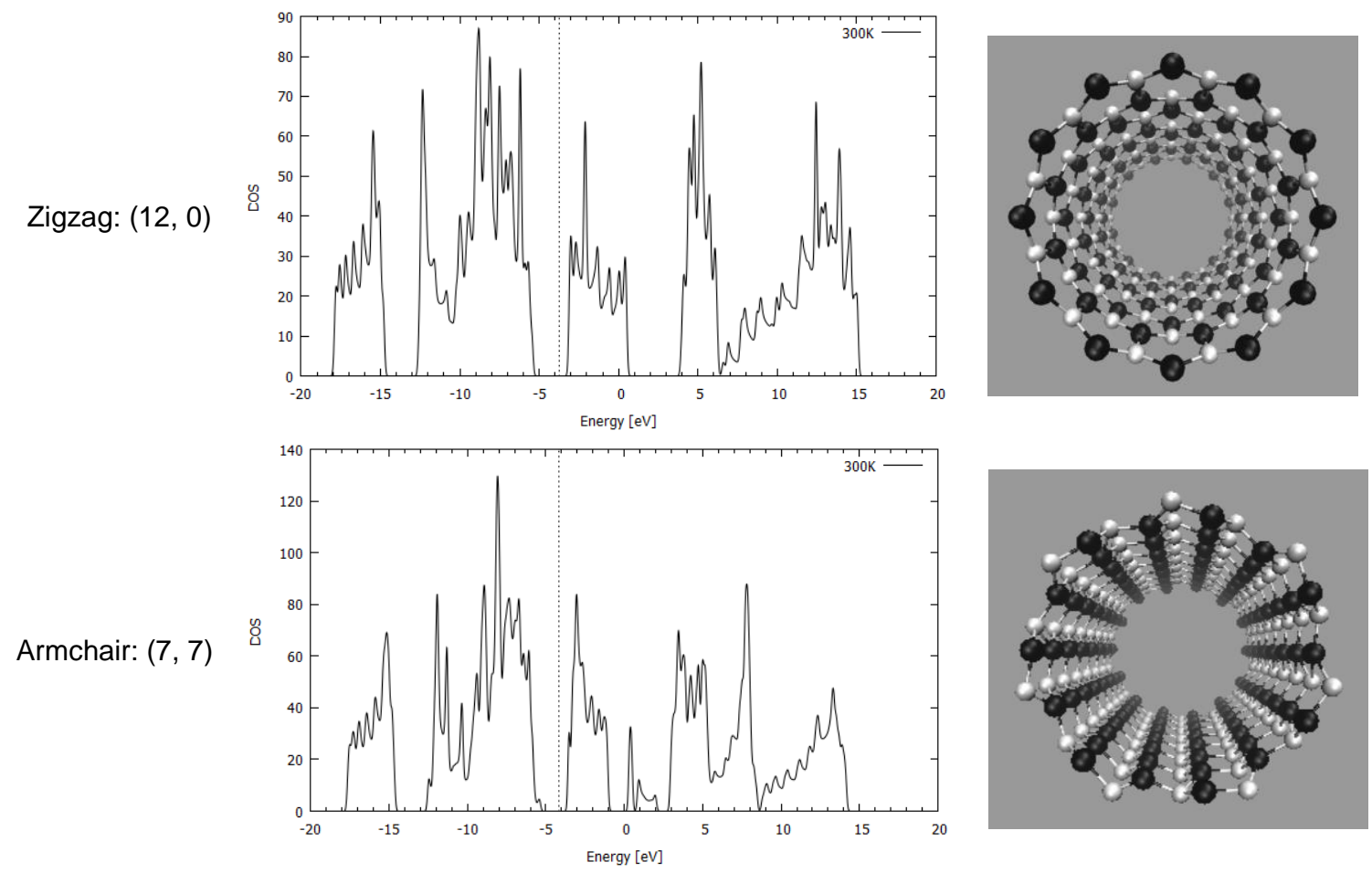

Fig. 1: Examples of SiCNT's; DOS and snapshots at 300K. The white and black atoms represent C and Si, respectively. The Fermi level $\left(E_{F}\right)$ is denoted by the dashed line.

Examples of the density of states (DOS) are shown in Fig. 1 for zigzag-type (12,0) and armchair type (7,7) SiCNT's; both types are insulator with a narrow band gap. We compare the DOS in Fig. 2 among the $(8,0)$ SiCNT, bulk SiC crystal, and the hydrogenated amorphous silicon sample [7], which indicates that the SiCNT has much narrower band gap than the bulk crystal, suggesting a good semi-conducting property (high conductivity) of SiCNT's.

For further investigation, size (circumference) dependence of the band gap is shown in Fig. 3 both for the zigzag types and armchair ones. The band gap becomes wider for thicker tubes. Armchair types have slightly narrower gaps than zigzag

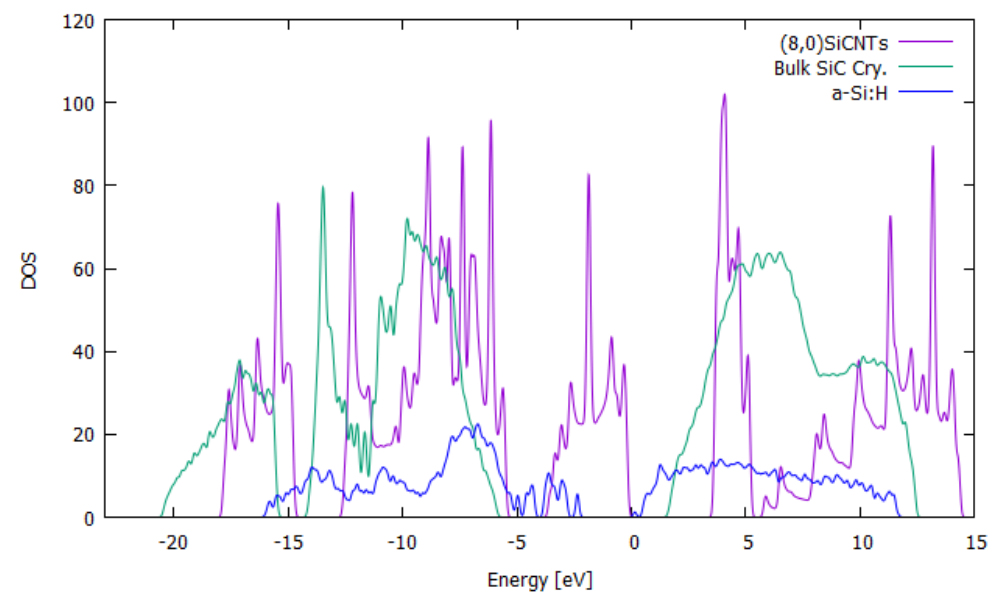

Fig. 2: Comparison of DOS at 300K among $(8,0) \mathrm{SiCNT}$, bulk SiC crystal, and hydrogenated amorphous silicon. 


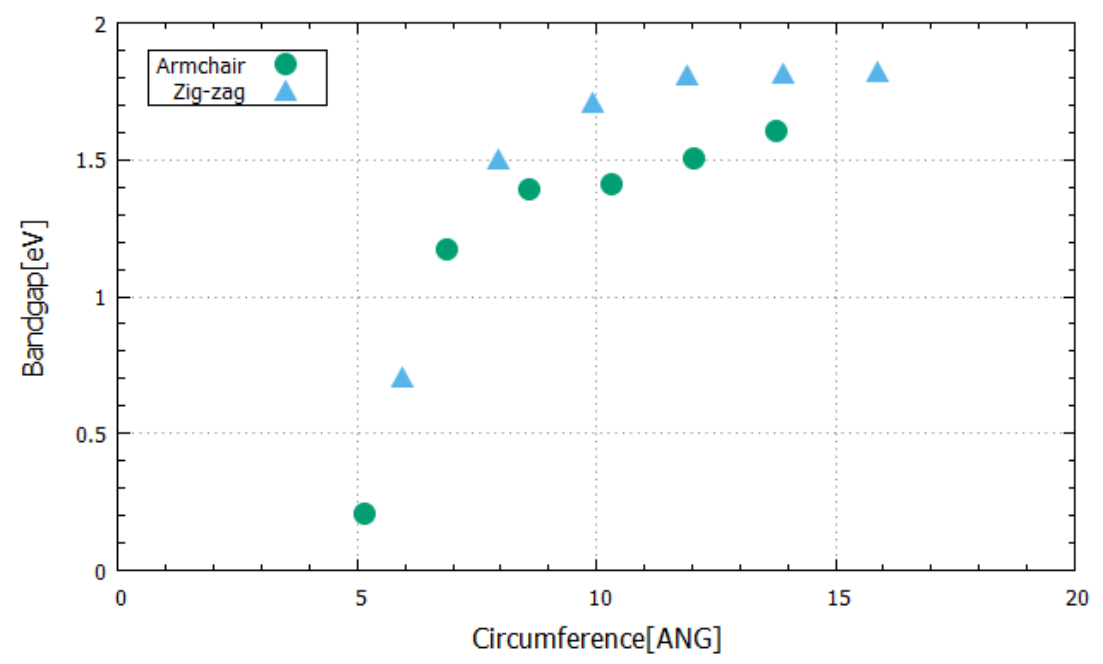

Fig. 3: Size dependence of the band gap.

ones. It is interesting to note that all of the SiCNT's are semiconductors, in contrast to CNT's, in which armchair types are metallic.

Examples of the band structure along the tube axis are shown in Fig. 4; apparently the zigzag types have a direct band gap while the armchair types have only an indirect one. No direct band gaps are reported for bulk SiC crystal, suggesting the possibility of SiCNT's for light-emitting devices.

We have also investigated the electronic transport properties. An example is shown in Fig.5, where both ends of a $(12,0)$ SiCNT are contacted with metallic electrodes (aluminum fcc crystal) and the transmission coefficient is evaluated based on the non-equilibrium Green's function method [6]. There exists a significant transmission around the Fermi level, which suggests the electronic states around the Fermi level have relevant contribution to the total current through the tube.

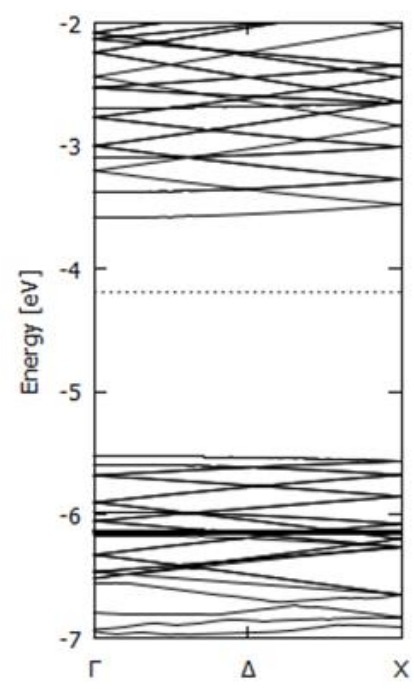

(a)

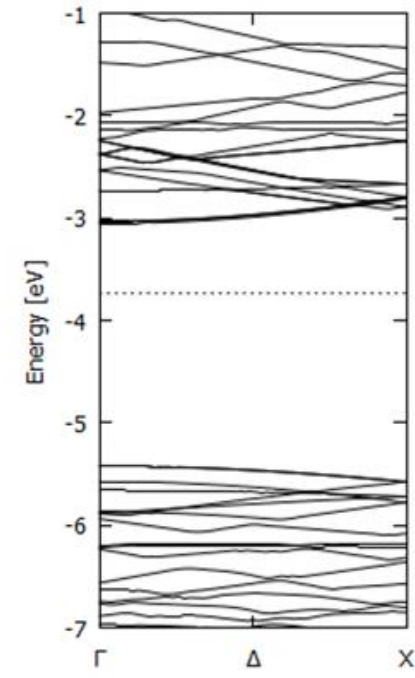

(b)

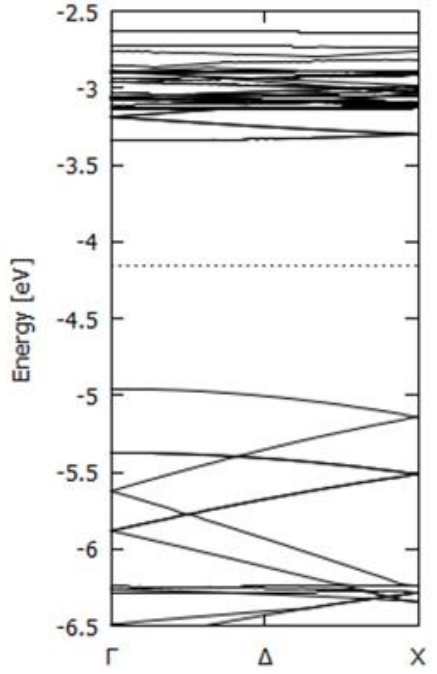

(c)

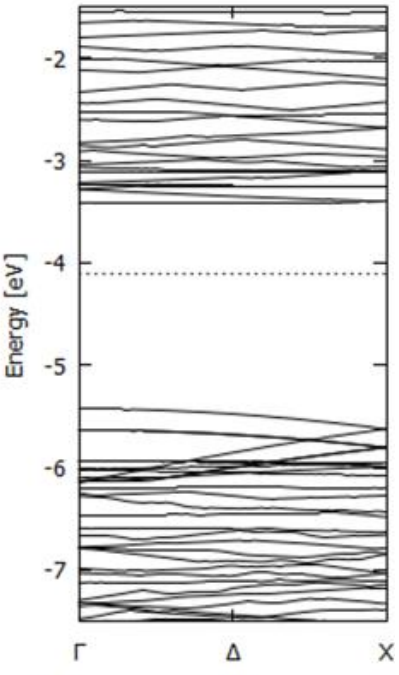

(d)

$(6,6)$

Fig. 4: Comparison of the band structure along the tube axis; (a) and (b) zigzag types, (c) and (d) armchair types. The Fermi level is denoted by dashed lines. 

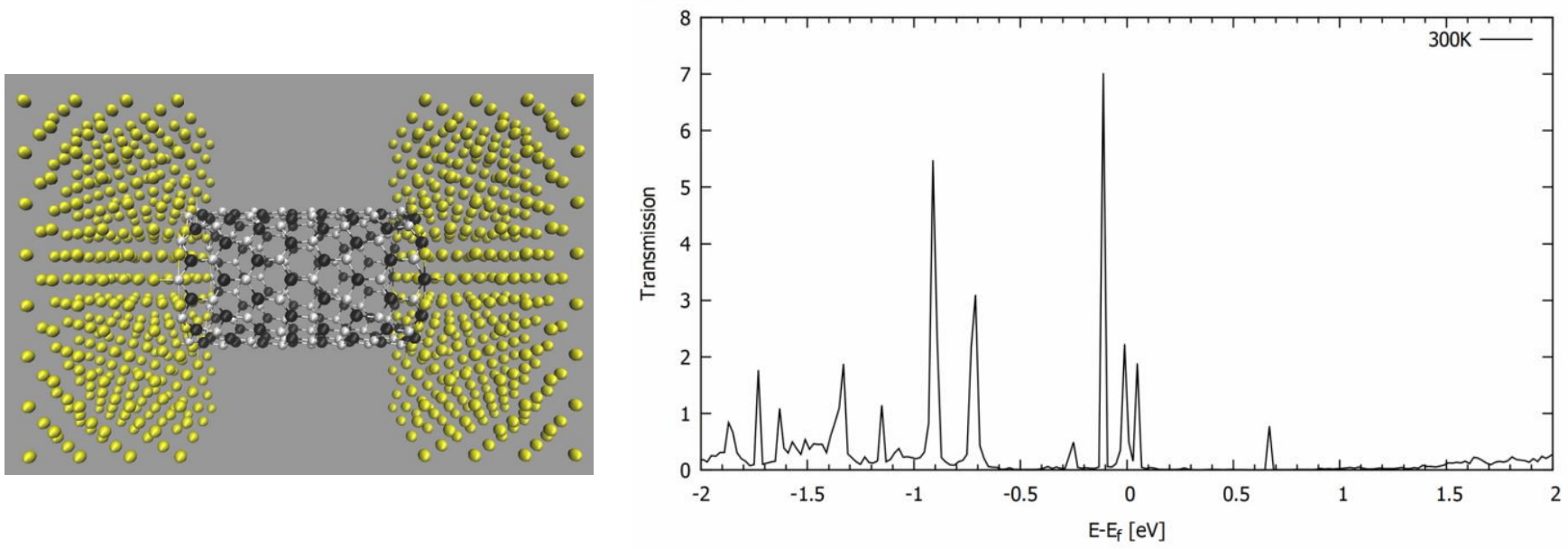

Fig. 5: Investigation of electron transport properties. (Left) system setup, where a single (12,0) SiCNT is sandwiched between aluminum electrode (Si: black, C: white, Al: yellow). (Right) obtained the transmission coefficient.

\section{Conclusion}

Quantum simulations based on the DFTB model were performed to investigate the electronic properties of various types of silicon carbide nanotubes. SiCNT's generally possess a narrower band gap than their bulk crystal, depending on their thickness. In particular, the band gap of armchair types is narrower than that of zigzag types. Direct band gap exists in zigzag type SiCNT's. The electron transport calculations suggest that the electronic states around the Fermi level should have major contribution to the total current. These findings are useful for designing various types of SiCNT devices, such as light emitting diodes and molecular transistors.

\section{References}

[1] N.G. Chopra, R.J. Luyken, K. Cherry, V.H. Crespi, M.L. Cohen, S.G. Louie, and A. Zettl, "Boron-nitride nanotubes," Science, 269, 966-967 (1995).

[2] A. Loiseau, F. Willaime, N. Demoncy, G. Hug, and H. Pascard, "Boron nitride nanotubes with reduced numbers of layers synthesized by arc discharge," Phys. Rev. Lett., 76, 4737-4740 (1996).

[3] D. Goldberg, Y. Bando, W. Han, K. Kurashima, and T. Sato, "Single-walled B-doped carbon, B/N-doped carbon and BN nanotubes synthesized from single-walled carbon nanotubes through a substitution reaction," Chem. Phys. Lett. 308, 337-342 (1999).

[4] F.S. Khan and J.Q. Broughton, "Relaxation of icosahedral-cage silicon clusters via tight-binding molecular dynamics," Phys. Rev. B, 43, 11754-11761 (1991).

[5] M. Menon, E. Richter, A. Mavrandonakis, G. Froudakis, and A.N. Andriotis, "Structure and stability of SiC nanotubes," Phys. Rev. B, 69, 115322 (2004).

[6] http://www.dftbplus.org/about-dftb/

[7] H. Li and M. Matsumoto, "Structure and electronic properties of a-Si:H investigated with quantum simulation," Proc. TANN'20 (2020). 